\title{
Symmetrical waveguide devices fabricated by direct UV writing
}

\author{
Færch, Kjartan Ullitz; Svalgaard, Mikael
}

Published in:

I E E E Photonics Technology Letters

Link to article, DOI:

10.1109/68.980496

Publication date:

2002

Document Version

Publisher's PDF, also known as Version of record

Link back to DTU Orbit

Citation (APA):

Færch, K. U., \& Svalgaard, M. (2002). Symmetrical waveguide devices fabricated by direct UV writing. I E E E Photonics Technology Letters, 14(2), 173-175. https://doi.org/10.1109/68.980496

\section{General rights}

Copyright and moral rights for the publications made accessible in the public portal are retained by the authors and/or other copyright owners and it is a condition of accessing publications that users recognise and abide by the legal requirements associated with these rights.

- Users may download and print one copy of any publication from the public portal for the purpose of private study or research.

- You may not further distribute the material or use it for any profit-making activity or commercial gain

- You may freely distribute the URL identifying the publication in the public portal

If you believe that this document breaches copyright please contact us providing details, and we will remove access to the work immediately and investigate your claim. 


\title{
Symmetrical Waveguide Devices Fabricated by Direct UV Writing
}

\author{
Kjartan Færch and Mikael Svalgaard
}

\begin{abstract}
Power splitters and directional couplers fabricated by direct UV writing in index matched silica-on-silicon samples can suffer from an asymmetrical device performance, even though the UV writing is carried out in a symmetrical fashion. This effect originates from a reduced photosensitivity in the vicinity of previous exposed areas. The imbalance can be counteracted by an appropriate reduction of the applied scan velocity in areas, where a previous scan has been carried out nearby.
\end{abstract}

Index Terms-Optical devices, optical materials, optical waveguide components, optical waveguides.

\section{INTRODUCTION}

D IRECT UV writing is a technique for fabrication of buried channel waveguides, where a focused ultraviolet (UV) laser beam is scanned across a silica-based sample [1]. The sample has a germanium-doped core layer in which the photosensitivity has been enhanced by indiffusion of deuterium $\left(D_{2}\right)$, enabling a UV-induced refractive index change of several times $10^{-3}$ [2]. Basic waveguide devices, such as power splitters and directional couplers, have been demonstrated with direct UV writing [3]. Circular guided mode profiles can be achieved by codoping the core layer with boron, thereby matching the unexposed core layer index to that of the surrounding buffer and cladding layers [4]. However, as reported here, in such index-matched samples, it has proven difficult to achieve splitters and couplers with the transmitted power distributed evenly between the output arms. We show that this asymmetry is due to the photosensitivity decreasing slightly in the vicinity of the first scan. We will present a simple method for counteracting this effect to achieve a symmetrical device performance, as well as an investigation of the physical manner in which subsequent scans affect each other.

\section{EXPERIMENTAL}

\section{A. Sample Structure and Waveguide Fabrication}

The samples consist of a silica-based three-layer structure (buffer, core, cladding) deposited by plasma-enhanced vapor deposition on a silicon substrate. The buffer/core/cladding thickness is $16 / 5.4 / 12 \mu \mathrm{m}$. The core layer is codoped with germanium and boron in a relative proportion, so that the refractive index is matched to that of the cladding and buffer. The intrinsic photosensitivity of the core is quite low, and has thus, been enhanced by loading with 2.9 mole $\% \mathrm{D}_{2}$ prior to the UV exposure.

Manuscript received June 19, 2001; revised October 10, 2001.

The authors are with the COM Center, Technical University of Denmark, Lyngby, DK-2800 Denmark (e-mail: kf@com.dtu.dk; svlgrd@com.dtu.dk).

Publisher Item Identifier S 1041-1135(02)00259-8.
Waveguides are fabricated by scanning the sample under a 257-nm continuous-wave beam with a power of $45 \mathrm{~mW}$. The $\mathrm{UV}$ beam is focused to a measured $1 / \mathrm{e}^{2}$ diameter of $3.1 \mu \mathrm{m}$. As shown later, the UV-induced refractive index change in indexmatched samples decreases significantly with the $\mathrm{D}_{2}$ concentration, hence, it is necessary to slow the rate of $\mathrm{D}_{2}$ outdiffusion by cooling the sample [5]. The sample is, therefore, mounted on a vacuum chuck, which is thermoelectrically cooled to $-35{ }^{\circ} \mathrm{C}$ in an $\mathrm{N}_{2}$ atmosphere (to prevent ice formation). After UV writing, the sample is annealed at $80{ }^{\circ} \mathrm{C}$ for $24 \mathrm{~h}$ to remove residual $\mathrm{D}_{2}$.

\section{B. Waveguide Characterization}

The waveguides and devices are characterized by measuring the insertion loss (averaged for all polarization states) and polarization-dependent loss (PDL), by butt-coupling standard single-mode optical fibers excited with a polarized $1.55-\mu \mathrm{m}$ laser. The effective index may be measured by inscripting a weak Bragg grating $(<0.1-\mathrm{dB}$ transmission loss $)$ in the waveguides. Finally, the waveguide width can be measured to an accuracy of $\pm 1 \%$ with a high-resolution optical microscope in combination with a charged-coupled device (CCD) camera [6].

For 1-cm-long straight waveguides, the measured insertion loss is typically $0.4 \mathrm{~dB}$, with a PDL of $0.15 \mathrm{~dB}$. The waveguides are $6.5 \mu \mathrm{m}$ wide, which is roughly a factor of two larger than the measured UV spot size. This suggests that the index change process is saturated in the central part of the exposed area. By comparing effective index and width measurements with mode field calculations, we have derived a step index value of $\sim 0.007$ for the fabricated waveguides [6]. The waveguides are robustly single mode at wavelengths in the $1.5-\mu \mathrm{m}$ band. All splitters and couplers presented in this letter have an excess loss of 0.05-0.2 $\mathrm{dB}$ and negligible excess PDL.

\section{Layout of Splitters and Couplers}

The fabricated splitters consist of three sections: an input arm, a lower output arm, and an upper output arm. The sections are scanned sequentially, starting with the input arm, followed by the upper output arm, and finally the lower output arm. Each scan starts at a central branching point and moves outwards, as indicated by arrows on the schematic overview in Fig. 1.

Each output arm starts with an $800-\mu \mathrm{m}$ straight section, tilted $0.2^{\circ}$ from the input arm, followed by circular arc s-bends (curvature radius of $35 \mathrm{~mm}$ ). The scan velocity applied for both output arms is $200 \mu \mathrm{m} / \mathrm{s}$. The output arm spacing is $81 \mu \mathrm{m}$ and the total device length is roughly $3 \mathrm{~mm}$.

The fabricated directional couplers have dual waveguide input/output ports, connected to a central coupling region 


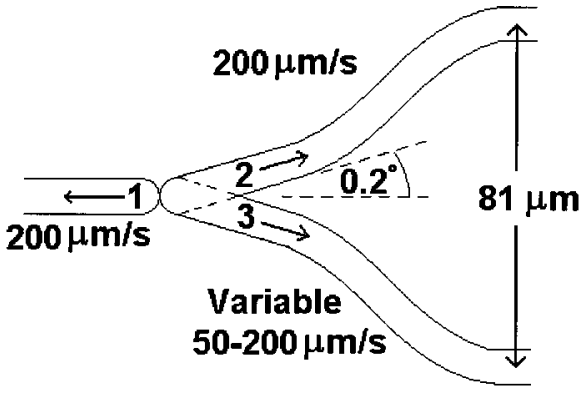

Fig. 1. Schematic layout of the splitter structure.

by $s$-bends. The scan velocity, radius of curvature, and port spacing is identical to that of the splitters described above. The couplers are written in two sequential scans, starting at the same side of the optical chip.

\section{DEVICE ASYMMETRY}

\section{A. Asymmetry of Splitters and Directional Couplers}

Note that the device layouts described above are completely symmetrical around the longitudinal axis. However, measurements show that the fabricated splitters exhibit a splitting ratio of $\sim 0.70$ (the relative amount of transmitted power contained in the first written output arm). As the measured widths after the branching point are identical for both output waveguides, this asymmetry must be due to a lower refractive index of the arm written in the second scan.

A similar asymmetric behavior is seen for the fabricated couplers. A center-to-center spacing in the central coupling region of $9 \mu \mathrm{m}$ was chosen as starting point. Varying the length of the central coupling region resulted in the coupling ratio varying in a squared sinusiodal fashion. This confirms that the simple equations for two coupled modes were applicable. The coupling ratio first peaked for a length of the central coupling region of $800 \mu \mathrm{m}$. However, the maximum value was not unity, as expected for a phase-matched coupler, but rather 0.95. No difference between the waveguide widths in the coupling region have been observed, hence, this behavior must also be due to a difference in refractive index between the two arms. For a larger waveguide spacing, the maximum coupling ratio increased, converging toward a value close to unity $(>0.999)$ for a center-to-center spacing of $\sim 12 \mu \mathrm{m}$. Hence, the range over which subsequent scans affect each other is roughly $12 \mu \mathrm{m}$. Due to similar fabrication methods for both splitters and couplers, the induced asymmetry is most likely caused by the same mechanism.

Simple analysis by a beam propagation method (BPM) of the actual splitter and coupler structures, estimates a refractive index difference of a few times $10^{-4}$ to account for the observed asymmetry.

\section{B. Correcting the Induced Asymmetry}

A nonsymmetrical power distribution is undesirable in many situations, such as interferometric applications and $1 \times \mathrm{N}$ power splitting. We have, therefore, applied a simple method for counter balancing the observed asymmetry. This method is based on the experience that the induced index change is easily

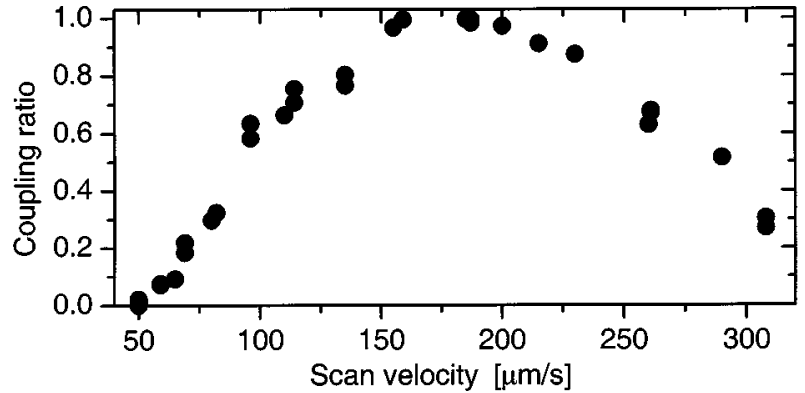

(a)

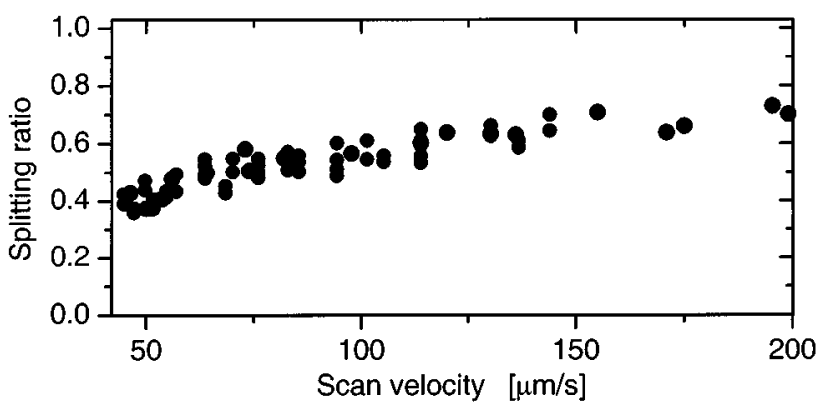

(b)

Fig. 2. (a) Coupling ratio and (b) splitting ratio versus the scan velocity in the second output arm. The scan velocity in the first output arm is fixed at $200 \mu \mathrm{m} / \mathrm{s}$.

controlled by varying the applied scan velocity [6]. Thus, by appropriately lowering the scan velocity for the second output arm, the index of the two closely spaced waveguides can be matched. This is illustrated for couplers in Fig. 2(a). Here, a series of couplers were written with a center-to-center spacing of $9 \mu \mathrm{m}$ and a coupling region length of $800 \mu \mathrm{m}$. With the scan velocity in the first arm being $200 \mu \mathrm{m} / \mathrm{s}$, the scan velocity of the second arm has to be lowered to $\sim 175 \mu \mathrm{m} / \mathrm{s}$ to obtain full cross coupling, and hence, identical waveguides. Implementing a similar method for the splitter fabrication resulted in the measurements in Fig. 2(b). Here, symmetrical splitting is achieved for a scan velocity in the second arm of $\sim 70 \mu \mathrm{m} / \mathrm{s}$. From effective index measurements on isolated waveguides [6], we have seen that such reductions in scan velocity results in several times $10^{-4}$ higher index change. This is in good agreement with our previous estimation of the index asymmetry using BPM calculations.

From several UV writing sessions over a period of more than half a year, we have verified that the optimization described above is quite stable without the need for readjustment. In addition, no penalty in terms of higher loss has been observed as a consequence of our asymmetry balancing. Hence, with this method, we are able to reliably fabricate symmetrical couplers and splitters, simply by appropriately adjusting the scan velocity applied during the fabrication of the second arm.

\section{DISCUSSION}

The cause of the observed index asymmetry has been addressed by considering two different scenarios.

The first scenario assumes a halo of UV light surrounding the main spot on the sample, originating from aberrations in the optical system. Waveguides spaced within the extent of this halo 


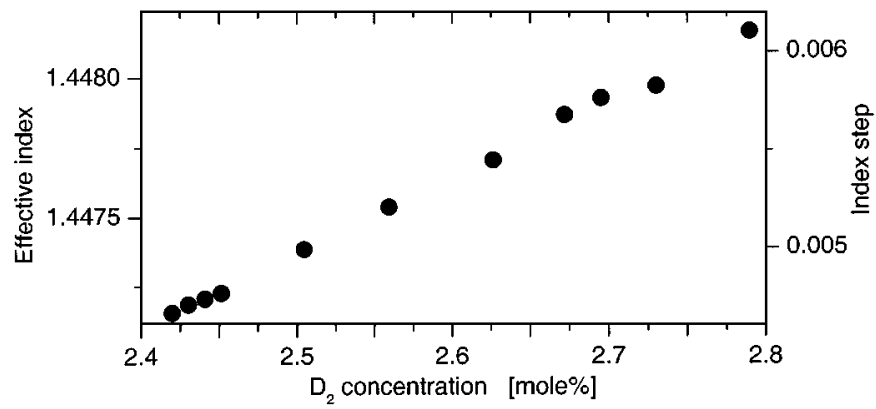

Fig. 3. Effective index and UV-induced step index versus the $\mathrm{D}_{2}$ concentration at the time of fabrication.

are either pre- or post-exposed to a weak field of UV radiation, depending on the order in which they are written. The observed index asymmetry could then arise from a nonsymmetrical response of such pre- or post-exposure. Intensity profile measurements of the focused beam have shown that the fabricated couplers will experience pre- and post-exposures with UV intensities $<10^{-3}$ times that of the UV spot center. A series of waveguides were written with either pre- or post-exposure, performed by scanning with a defocused beam of $10^{-3}$ times the intensity of the normal UV spot. However, no measurable difference in the effective index $\left(<3 \times 10^{-5}\right)$ or width $(<2 \%)$ was observed in the pre- and post exposed waveguides. Thus, any asymmetry toward pre-/post-exposure is at least one order of magnitude less than required to account for the observed device asymmetry.

The second scenario assumes that the high UV intensity of the central beam $\left(\sim 0.6 \mathrm{MW} / \mathrm{cm}^{2}\right)$ initiates some process in the nearby, unexposed glass, as it passes nearby which reduces the photosensitivity. It is quite difficult to test directly for this scenario, since we are not sure of the nature of the initiated process. However, it is likely that the photosensitivity reduction is coupled to a reduction in the concentration of $\mathrm{D}_{2}$, since essentially all of the photosensitivity is caused by $\mathrm{D}_{2}$ loading. To examine the effect of a reduced $\mathrm{D}_{2}$ concentration, a series of waveguides were written with four-minute intervals, during which $\mathrm{D}_{2}$ was allowed to outdiffuse at a temperature of $23{ }^{\circ} \mathrm{C}$. At this temperature, the 1/e outdiffusion time was later measured to be 16 $h$ [7], from which the $D_{2}$ concentration at the fabrication time of each waveguide could be determined. Measurements show that the waveguide width is constant over the sampled range, while the effective index decreases almost linearly for lower $\mathrm{D}_{2}$ concentrations (shown in Fig. 3). Also shown in Fig. 3 is the UV-induced index step, as derived using the techniques outlined in [6]. The index is seen to decrease by roughly $10^{-4}$ pr. percent reduction in $\mathrm{D}_{2}$ concentration. A few percent reduction of the $\mathrm{D}_{2}$ concentration in the vicinity of the first scan could, therefore, easily account for the observed device asymmetry. Such a reduction could be caused by a redistribution of $\mathrm{D}_{2}$ as it diffuses from the UV spot surroundings to the exposed area, where it is depleted in the index change process [7]. It could also be caused by localized outdiffusion through the buffer and cladding. Since the glass is cooled to $-35{ }^{\circ} \mathrm{C}$, either process requires a thermal heating due to the $\mathrm{UV}$ exposure. A $1 \% \mathrm{D}_{2}$ reduction occurring by outdiffusion/redistribution in the $\sim 10^{-1} \mathrm{~s}$ it takes the UV spot to pass, would require a temperature increase of several hundred Kelvin [8]. Whether such heating actually occurs is currently being investigated in detail.

A mechanism involving local changes in the $\mathrm{D}_{2}$ concentration is supported by the fact that device asymmetry problems were not experienced in nonindex matched samples [3], where the UV-induced index change depends less strongly on the $\mathrm{D}_{2}$ concentration.

\section{CONCLUSION}

Splitters and couplers fabricated by direct UV writing in $\mathrm{D}_{2}$ loaded germanium/boron doped silica glass can exhibit a significantly asymmetric performance, even though the UV writing is carried out in a symmetrical fashion. We have shown that this effect is due to a photosensitivity reduction in the glass material surrounding a previously exposed area. The refractive index difference for two closely spaced waveguides can be as large as a few times $10^{-4}$. Symmetrical devices can be fabricated by reducing the scan velocity used in the second device arm, hereby counteracting the photosensitivity reduction caused by the first scan. Our measurements suggest that subsequent scans affect each other by causing a localized reduction in the $\mathrm{D}_{2}$ concentration of a few percent.

\section{REFERENCES}

[1] M. Svalgaard, C. V. Poulsen, A. Bjarklev, and O. Poulsen, "Direct UV-writing of buried single mode channel waveguides in Ge-doped silica films," Electron. Lett., vol. 30, no. 17, pp. 1401-1403, 1994.

[2] P. J. Lemaire, R. M. Atkins, V. Mizrahi, and W. A. Reed, "High pressure $\mathrm{H}_{2}$ loading as a technique for achieving ultrahigh UV photosensitivity and thermal sensitivity in $\mathrm{GeO}_{2}$ doped optical fibers," Electron. Lett., vol. 29, no. 13, pp. 1191-1193, 1993.

[3] M. Svalgaard, "Direct writing of planar waveguide power splitters and directional couplers using a focused ultraviolet laser beam," Electron. Lett., vol. 33, no. 20, pp. 1694-1695, 1997.

[4] D. Zauner, K. Kulstad, J. Rathje, and M. Svalgaard, "Directly UV-written silica-on-silicon planar waveguides with low insertion loss," Electron. Lett., vol. 34, no. 16, pp. 1582-1584, 1998.

[5] M. Svalgaard, "Effect of $\mathrm{D}_{2}$ outdiffusion on direct UV writing of optical waveguides," Electron. Lett., vol. 35, no. 21, pp. 1840-1842, 1999.

[6] M. Svalgaard, A. Harpøth, and L. Leick, "Experimental model for the waveguiding properties of directly UV written waveguides," in Optical Society Amer. Technical Dig. Series: Bragg Gratings, Photosensitivity and Poling in Glass Waveguides, 2001.

[7] B. Malo, J. Albert, K. O. Hill, F. Bilodeau, and D. C. Johnson, "Effective index drift from molecular hydrogen diffusion in hydrogen-loaded optical fibers and its effect on Bragg grating fabrication," Electron. Lett.,, vol. 30, no. 5, pp. 442-444, 1994.

[8] P. J. Lemaire, "Reliability of optical fibers exposed to hydrogen: Prediction of long-term loss increases," Opt. Eng., vol. 30, no. 6, pp. 780-789, 1991. 